Tohoku J. Exp. Med., 2003, 201, 201-211

\title{
Changes in Plasma Lactate and Pyruvate Concentrations after Taking a Bath in Hot Deep Seawater
}

\author{
Yasuo Tsuchiya, Tominiro Shimizu, ${ }^{1}$ Teruyuki Tazawa, ${ }^{2}$ Norio Shibuya, ${ }^{3}$ \\ Kazutoshi Nakamura and Masaharu Yamamoto \\ Department of Community Preventive Medicine, Niigata University \\ Graduate School of Medical and Dental Sciences, Niigata 951-8510, \\ ${ }^{1}$ Department of Sports and Health Sciences, Joetsu University of Education, \\ Joetsu 943-8512, \\ ${ }^{2}$ Aquatic Health Association, Niigata 950-0912, and \\ ${ }^{3}$ Niigata Collage of Medical Technology, Niigata 950-2076
}

Tsuchiya, Y., Shimizu, T., Tazawa, T., Shibuya, N., Nakamura, K. and Yamamoto, M. Changes in Plasma Lactate and Pyruvate Concentrations after Taking a Bath in Hot Deep Seawater. Tohoku J. Exp. Med., 2003, 201 (4), 201-211 - The use of deep seawater (DSW) in thalassotherapy has begun in Japan. To clarify the health effects of DSW on the human body, we investigated the changes in plasma lactate and pyruvate concentrations, or subjective judgment scores, after bathing at rest in 9 healthy young men. Subjects were immersed for 10 minutes in DSW, surface seawater (SSW), and tap water (TW) heated to $42^{\circ} \mathrm{C}$. Plasma samples were collected before bathing, immediately after bathing, and 60 minutes after bathing. The scores were obtained by an oral comprehension test. In the DSW bathing, plasma lactate and pyruvate concentrations showed no significant changes immediately after bathing or 60 minutes after bathing. In contrast, subjects who bathed in SSW showed a significant decrease in lactate concentrations 60 minutes after bathing compared with immediately after bathing. Subjects who bathed in TW showed a significant increase in lactate concentrations immediately after bathing compared with before bathing, and they showed a significant decrease in lactate and pyruvate concentrations 60 minutes after bathing. We found no significant change in the thermal sensation score in the DSW bathing, though significant differences were found between before and immediately after bathing in the SSW and TW groups. Moreover, the score decreased significantly 60 minutes after bathing compared to immediately after bathing in the TW bathing. Higher concentrations of salts contained DSW such as sodium, nitrate-nitrogen, phosphate-phosphorus, and silicate-silicon may have a

Received August 8, 2003; revision accepted for publication October 14, 2003.

Address for reprints: Yasuo Tsuchiya, Division of Social and Environmental Medicine, Department of Community Preventive Medicine, Niigata University Graduate School of Medical and Dental Sciences, 1-757 Asahimachi-dori, Niigata 951-8510, Japan.

e-mail: troof@med.niigata-u.ac.jp 
good influence on human health. Although additional studies are needed to support our findings, DSW is the mildest water to the human body among the three kinds of water, since no significant changes in the items measured were found only in DSW.— deep seawater; hot water bathing; plasma lactate and pyruvate; subjective judgment

(C) 2003 Tohoku University Medical Press

We have been conducting research on the health effects of deep seawater (DSW) collected from the Japan Sea. This water is called Japan Sea Proper Water. First, we have demonstrated that the administration of diluted DSW to mice caused increased serum IgG and glucose values, as compared with values obtained after administration of purified water (Tsuchiya et al. 2002). Second, we have conducted experiments to clarify the association between hot DSW bathing and changes in the immune cell distribution of peripheral blood (unpublished). As a series of experiments to clarify the characteristics of DSW, the present study was carried out to examine the changes in plasma lactate and pyruvate concentrations, and the subjective judgment scores after taking a bath in DSW as compared with the effects of bathing in surface seawater (SSW) or tap water (TW).

In general, hot spring bathing is useful for maintaining hygiene, improving blood circulation, and mitigating muscular fatigue and pain because of the water's warm temperature, pressure, and buoyancy effects (Ueda 2001). Recently, the use of DSW in thalassotherapy has begun in Japan. The efficacy of seawater, sea mud, and seaweed for the treatment of some diseases has been shown in previous studies (Tsubaki et al. 1994; Nissen et al. 1998; Harari et al. 2000). Moreover, some studies regarding the effects of balneotherapy at the Dead Sea have been published (Sukenik et al. 1994; Elkayam et al. 2000; Buskila et al. 2001; Neumann et al. 2001; Evcik et al. 2002). On the other hand, an association between hot water bathing and claimed subjective fatigue has pre- viously been observed (Kiuchi et al. 1983). In other words, conflicting information has been gathered regarding the health effects of hot water bathing.

Seawater and human serum are of similar composition (Linder 1991). In addition, DSW is very pure, is rich in several types of inorganic nutrient salts, and has low temperature stability (Sasaki 2001). We therefore hypothesized that DSW would be beneficial to the human body. To our knowledge, however, no study has been published regarding the health effects of hot DSW bathing. The present study was initiated to clarify the changes in blood examination values and subjective judgment scores caused by taking a hot DSW bath. More specifically, we examined the changes in plasma lactate and pyruvate concentrations, and in the scores of thermal sensation, the degree of fatigue, and the comfort level after hot DSW bathing.

The reasons why lactate and pyruvate concentrations are taken into consideration are as follows. First, it is known that repeated hot spring bathing causes a decrease in blood lactate concentrations (Yamana et al. 2002), and that the decreased lactate concentrations can relieve fatigue. The change of plasma lactate concentration after bathing may be able to evaluate the degree of recovery from fatigue. Second, Lactate and pyruvate are produced in the glycolytic pathway. Lactate is produced by anaerobic glycolysis, and pyruvate is oxidized aerobically in mitochondrion. These concentrations are used to evaluate the strenuousness of exercise or the degree of fatigue (Eastham 1985). The changes of plasma lactate and pyruvate concentrations may be 
able to evaluate the degree of fatigue after bathing.

The aim of the present study was to clarify the changes in plasma lactate and pyruvate concentrations, or the subjective judgment scores after hot DSW bathing without exercise tolerance. The results regarding DSW bathing were compared with those in SSW and TW bathing.

\section{SUBJECTS AND METHODS}

Subjects

A total of 9 healthy male students with a mean age of 19.3 years (S.D., 1.0), a mean height of $170.7 \mathrm{~cm}$ (S.D., 3.3), and a mean weight of $61.9 \mathrm{Kg}$ (S.D., 10.4) agreed to participate in this study. Written informed consent was obtained from each subject immediately after they were informed of the purpose of the study and the potential risks involved, including fluctuations in blood pressure after bathing, and blood collection three times daily. The subjects were asked to take their last meal at least 12 hours before participating in this study. The Ethics Committee at Niigata University School of Medicine approved our methods.

\section{Collection of DSW and SSW}

DSW and SSW were collected from respective depths of approximately 300 meters and 10 meters in the Japan Sea $\left(37.50-38.00^{\circ} \mathrm{N}\right.$ latitude; $138.30-138.45^{\circ} \mathrm{N}$ longitude), using the DSWdrawing system developed jointly by HONMA Co., Ltd., and KITAC Corporation (Niigata). Water was collected in plastic containers and sent immediately to our laboratory. The water was used within 5 days.

\section{Experimental procedure}

This study was performed using three kinds of water and two bathtubs for three consecutive days; the experiment, which took place between 8:00 a.m. and 12:00 a.m., was repeated five times daily in July 2002. After two different types of waters were poured into the two bathtubs, two subjects were bathed in numeric order. The subjects were not told which type of water was used and were tested at the same time for three consecutive days to avoid the influence of physical conditions or the circadian rhythms of leukocytes (Abo et al. 1981).

The experimental procedure was as follows: two experimental bathtubs $(1.0 \times 0.6 \times 0.5$ $m$ depth) made of fiber-reinforced plastic were set up in a prefabricated bathroom $(2.1 \times 5.3 \times 2.3$ $\mathrm{m}$ height), where the temperature was maintained at $32 \pm 1^{\circ} \mathrm{C}$ and the relative humidity was kept at $70 \pm 1 \%$ during the experiment.

The subjects entered a prefabricated waiting room $(2.1 \times 5.3 \times 2.3 \mathrm{~m}$ height $)$, where the temperature was maintained at $27 \pm 2^{\circ} \mathrm{C}$ and the relative humidity was kept at $56 \pm 2 \%$. Here, subjects rested for 30 minutes before the start of the experiment. The first blood sample $(4 \mathrm{ml})$ was collected from the median cubital vein of each subject. Thermal sensation, the degree of fatigue, and comfort level were recorded at that time. Thermal sensation was evaluated using the following categories (Houghton and Yagloglou 1923; Yoshida 1978): (1) cold; (2) cool; (3) slightly cool; (4) neutral; (5) slightly warm; (6) warm; (7) hot; (8) very hot. The degree of fatigue (Nakamura et al. 1996) and comfort level (Winslow et al. 1937; Hardy 1970) were evaluated as follows: for the degree of fatigue, (1) not tired; (2) hardly tired; (3) slightly tired; (4) tired; (5) very tired; for the comfort level, (1) very pleasant; (2) pleasant; (3) indifferent; (4) unpleasant; (5) very unpleasant.

Water was poured into the bathtub. The water temperature was set at $42^{\circ} \mathrm{C}$ at the start of the experiment and was regulated by a temperature-control device throughout the experiment. The subject moved from the waiting room to the bathroom and immersed himself in the bathtub with his legs extending forward. The surface of the water in the bathtub reached the level of the diaphragm. The bathing continued for 10 minutes. After bathing for 10 
minutes, the subject left the bathtub and moved to a prefabricated resting room $(2.1 \times 5.3 \times 2.3 \mathrm{~m}$ height) where the temperature $\left(26 \pm 1^{\circ} \mathrm{C}\right)$ and the relative humidity $(55 \pm 1 \%)$ were controlled, the latter by an air conditioner. The subject rested immediately on a reclining chair, at which time a second blood sample $(4 \mathrm{ml})$ was collected. Thermal sensation, the degree of fatigue, and comfort level were recorded at that time. After drying themselves thoroughly with a towel, the subjects moved to the waiting room and dressed, then rested and relaxed there until the third blood collection $(4 \mathrm{ml})$ was performed 60 minutes after bathing. At this point, each subject was again asked to report his subjective judgments.

\section{Analysis of blood samples}

Approximately $2 \mathrm{ml}$ of blood sample was transferred into an anti-coagulation tube containing NaF and EDTA-2K for the determination of lactate and pyruvate concentrations. The sample was centrifuged at $3000 \mathrm{rpm}$ for 2 minutes immediately after mixing the blood with the anti-coagulants. Plasma was separated immediately after the centrifugation and stored at minus $80^{\circ} \mathrm{C}$ until determination. An automatic analyzer (Clinical Analyzer 7150, Hitachi High-Technologies Corporation,
Tokyo) was used for the determination of plasma lactate and pyruvate concentrations. Reagents (Determiner LA, Determiner PA) and calibrators were purchased from Kyowa Medex Co., Ltd. (Tokyo).

The residual blood sample was transferred into an anti-coagulation tube containing EDTA$2 \mathrm{Na}$ for the determination of hemoglobin $(\mathrm{Hb})$ to evaluate the hemoconcentrations after bathing. An automated hematology analyzer (SF3000, Sysmex Corporation, Kobe) was used for the determination of $\mathrm{Hb}$.

\section{Statistical analysis}

All statistical procedures were performed using Statistical Analysis System software (SAS Institute Inc., Cary, NC, USA). To compare the mean, absolute, and relative values between repeated measurements, or among three water groups, we used repeated measures analysis of variance (ANOVA). Differences were considered significant when the $p$-value was less than 0.05 .

\section{RESULTS}

Table 1 shows the values of key parameters in Japan Sea proper Water. No evident differences between DSW and SSW were found other than SSW having higher values of water

Table 1. Values of key parameters in Japan Sea Proper Water

\begin{tabular}{lccc}
\hline Parameter & Unit & Deep seawater & Surface seawater \\
\hline Temperature & ${ }^{\circ} \mathrm{C}$ & 5.0 & 13.7 \\
$\mathrm{pH}$ & & 8.1 & 7.7 \\
Salinity & $\% 0$ & 34.1 & 33.2 \\
Dissolved oxygen & $\mathrm{mg} / \mathrm{liter}$ & 5.1 & 7.3 \\
Total organic carbor & $\mathrm{mg} / \mathrm{liter}$ & 1.8 & 2.0 \\
Nitrate $\left(\mathrm{NO}_{3}-\mathrm{N}\right)$ & $\mathrm{mg} / \mathrm{liter}$ & 1.29 & 0.11 \\
Phosphate $\left(\mathrm{PO}_{4}-\mathrm{P}\right)$ & $\mathrm{mg} / \mathrm{liter}$ & 0.18 & 0.05 \\
Silicate $\left(\mathrm{SiO}_{2}-\mathrm{Si}\right)$ & $\mathrm{mg} / \mathrm{liter}$ & 1.34 & 0.02 \\
Potassium & $\mathrm{mg} / \mathrm{liter}$ & 392 & 392 \\
Calcium & $\mathrm{mg} / \mathrm{liter}$ & 519 & 515 \\
Magnesium & $\mathrm{mg} /$ liter & 1420 & 1370 \\
Sodium & $\mathrm{mg} / \mathrm{liter}$ & 10361 & 9872 \\
Chloride & $\mathrm{mg} /$ liter & 19400 & 18800 \\
\hline
\end{tabular}


temperature and dissolved oxygen, while DSW had higher values of nitrate-nitrogen, phosphate-phosphorus, and silicate-silicon.

Table 2 shows the hot water bathinginduced changes in lactate, pyruvate and $\mathrm{Hb}$ concentrations. Plasma lactate concentrations showed no significant changes in the DSW bathing. However, the values increased significantly immediately after bathing compared to before bathing in TW $(p<0.01)$, and decreased significantly 60 minutes after bathing compared to immediately after bathing in the SSW ( $p<$ $0.05)$ and TW $(p<0.01)$ groups, though no significant differences were found among the three water groups under the three conditions.

Plasma pyruvate concentrations showed a significantly lower value 60 minutes after bathing compared with immediately after bathing in TW $(p<0.01)$. No significant differences were obtained in the lactate/pyruvate ratio in the present study.

$\mathrm{Hb}$ concentrations increased immediately after bathing in the three water groups compared with before bathing, though a significant difference was found in TW $(p<0.05)$, and then significant decreases were found between immediately after bathing and 60 minutes after bathing in the three water groups. Moreover, the values showed a significant decrease 60 minutes after bathing compared with before bathing in TW $(\phi<0.05)$. However, significant differences were observed between DSW and TW before and immediately after bathing $(\not<0.01)$ between SSW and TW under the three conditions $(p<0.01)$.

As shown in Table 3, the relative changes were calculated as the percentage of the value obtained before bathing to clarify the changes in

TABLE 2. Hot water bathing-induced changes in blood examination values

\begin{tabular}{|c|c|c|c|}
\hline & Before bathing & After bathing & 60 minutes after bathing \\
\hline \multicolumn{4}{|c|}{ Lactate (mmol/liter) } \\
\hline DSW & $0.85 \pm 0.18$ & $1.00 \pm 0.25$ & $0.79 \pm 0.30$ \\
\hline SSW & $0.86 \pm 0.18$ & $0.96 \pm 0.13$ & $0.73 \pm 0.21^{\dagger}$ \\
\hline TW & $0.83 \pm 0.15$ & $1.04 \pm 0.18^{* *}$ & $0.72 \pm 0.09^{\dagger^{\dagger}}$ \\
\hline \multicolumn{4}{|c|}{ Pyruvate (mmol/liter) } \\
\hline DSW & $0.064 \pm 0.019$ & $0.079 \pm 0.036$ & $0.057 \pm 0.020$ \\
\hline SSW & $0.064 \pm 0.012$ & $0.073 \pm 0.019$ & $0.056 \pm 0.023$ \\
\hline TW & $0.062 \pm 0.017$ & $0.075 \pm 0.027$ & $0.056 \pm 0.019^{\dagger \dagger}$ \\
\hline \multicolumn{4}{|c|}{ Lactate/Pyruvate ratio } \\
\hline DSW & $13.7 \pm 1.8$ & $14.5 \pm 5.4$ & $14.3 \pm 4.0$ \\
\hline SSW & $13.7 \pm 2.5$ & $14.6 \pm 5.7$ & $16.4 \pm 11.7$ \\
\hline TW & $13.9 \pm 3.5$ & $15.9 \pm 6.6$ & $14.4 \pm 5.1$ \\
\hline \multicolumn{4}{|c|}{ Hemoglobin (g/liter) } \\
\hline DSW & $1.53 \pm 0.11$ & $1.55 \pm 0.11$ & $1.51 \pm 0.12^{\dagger^{\dagger}}$ \\
\hline SSW & $1.52 \pm 0.12$ & $1.53 \pm 0.12$ & $1.50 \pm 0.12^{\dagger^{\dagger}}$ \\
\hline TW & $1.58 \pm 0.13$ & $1.61 \pm 0.13^{*}$ & $1.55 \pm 0.13^{*,+\dagger}$ \\
\hline
\end{tabular}

DSW, deep seawater; SSW, surface seawater; TW, tap water.

Each value represents mean \pm s.D. of 9 subjects.

Significant differences compared to the value before bathing are indicated by ${ }^{*} p<0.05,{ }^{* *} p<0.01$.

Significant differences compared to the value immediately after bathing are indicated by ${ }^{\dagger} p<0.05,{ }^{\dagger} p<0.01$. 
TABLE 3. Relative changes in lactate, pyruvate, and $\mathrm{Hb}$ concentrations

\begin{tabular}{cccc}
\hline & Before bathing & After bathing & 60 minutes after bathing \\
\hline Lactate & & & \\
DSW & 100 & $120 \pm 26$ & $91 \pm 27$ \\
SSW & 100 & $116 \pm 27$ & $86 \pm 28^{\dagger}$ \\
TW & 100 & $128 \pm 24^{* *}$ & $89 \pm 17^{\dagger+}$ \\
Pyruvate & & & \\
DSW & 100 & $128 \pm 51$ & $94 \pm 36$ \\
SSW & 100 & $114 \pm 29$ & $88 \pm 35$ \\
TW & 100 & $117 \pm 28$ & $89 \pm 18^{\dagger}$ \\
Hemoglobin & & & \\
DSW & 100 & $101 \pm 2$ & $99 \pm 2^{\dagger}$ \\
SSW & 100 & $101 \pm 2$ & $99 \pm 1^{\dagger+}$ \\
TW & 100 & $102 \pm 2^{*}$ & $98 \pm 2^{\dagger+}$ \\
\hline
\end{tabular}

DSW, deep seawater; SSW, surface seawater; TW, tap water.

Each value represents mean \pm s.D. of 9 subjects.

The relative changes were calculated as the percentage of the value obtained before bathing.

Significant differences compared to the value before bathing are indicated by ${ }^{*} p<0.05,{ }^{* *} p<0.01$.

Significant differences compared to the value immediately after bathing are indicated by ${ }^{\dagger} p<0.05,{ }^{\dagger \dagger} p<0.01$.

lactate, pyruvate, and $\mathrm{Hb}$ concentrations caused by hot water bathing because the initial values among the three water groups were different. Concentrations peaked after bathing and returned to the value before bathing in the three water groups.

Lactate concentrations increased $28 \%$ with statistical significance immediately after bathing compared with before bathing in TW $(p<$ 0.01 ), though the values in DSW and SSW increased $20 \%$ and $16 \%$, respectively, with no significances. Then, the concentrations in SSW and TW decreased 30\% $(p<0.05)$ and 39\% $(p<0.01)$ with statistical significance 60 minutes after bathing compared with immediately after bathing, respectively.

Pyruvate concentrations in TW decreased $26 \%$ with statistical significance 60 minutes after bathing compared to immediately after bathing, though no significant changes were observed in the DSW and SSW groups.

$\mathrm{Hb}$ concentrations in TW increased $2 \%$ with statistical significance immediately after bathing compared with before bathing, and the values in the three water groups decreased 2$4 \%$ with statistical significance. However, we observed no significant differences in the relative changes in $\mathrm{Hb}$ among the three water groups under the three conditions. Changes in lactate and pyruvate concentrations immediately after bathing and 60 minutes after bathing were greater than the changes in $\mathrm{Hb}$ concentrations.

Table 4 shows the changes in the subjective judgment scores. Thermal sensation scores showed significantly higher values immediately after bathing compared with before bathing in the SSW $(p<0.01)$ and TW $(p<0.01)$ groups, and a significantly lower value 60 minutes after bathing than after bathing in TW $(p<0.01)$. No significant changes in DSW were found between repeated measurements. However, significant differences were found between SSW and TW before and after bathing, and between 
TABLE 4. Changes in subjective judgment scores

\begin{tabular}{|c|c|c|c|}
\hline & Before bathing & After bathing & 60 minutes after bathing \\
\hline \multicolumn{4}{|c|}{ Thermal sensation } \\
\hline DSW & $3.89 \pm 0.87$ & $5.00 \pm 1.83$ & $3.56 \pm 0.96$ \\
\hline SSW & $3.44 \pm 0.68$ & $5.00 \pm 0.94^{* *}$ & $4.11 \pm 0.74$ \\
\hline TW & $4.89 \pm 1.20$ & $6.78 \pm 0.42^{* *}$ & $4.67 \pm 0.94^{\dagger \dagger}$ \\
\hline \multicolumn{4}{|c|}{ Degree of fatigue } \\
\hline DSW & $2.00 \pm 0.67$ & $2.44 \pm 0.50$ & $2.00 \pm 0.67$ \\
\hline SSW & $2.33 \pm 0.67$ & $2.56 \pm 0.68$ & $2.33 \pm 0.67$ \\
\hline TW & $2.89 \pm 0.31$ & $3.11 \pm 0.74$ & $2.67 \pm 0.67$ \\
\hline \multicolumn{4}{|c|}{ Comfort level } \\
\hline DSW & 3.00 & $2.56 \pm 0.68$ & $2.67 \pm 0.67$ \\
\hline SSW & $2.78 \pm 0.42$ & $2.78 \pm 0.42$ & $2.33 \pm 0.67$ \\
\hline TW & $3.22 \pm 0.63$ & $3.00 \pm 0.67$ & $2.67 \pm 0.47$ \\
\hline
\end{tabular}

DSW, deep seawater; SSW, surface seawater; TW, tap water. Each value represents mean \pm s.D. of 9 subjects.

Significant differences compared to the value before bathing are indicated by ${ }^{* *} p<0.01$.

Significant differences compared to the value immediately after bathing are indicated by ${ }^{\dagger \dagger} p<0.01$.

Scores indicate: for thermal sensation (1) cold; (2) cool; (3) slightly cool; (4) neutral; (5) slightly warm; (6) warm; (7) hot; (8) very hot, for the degree of fatigute (1) not tired; (2) hardly tired; (3) slightly tired; (4) tired; (5) very tired, for comfort level (1) very pleasant; (2) pleasant; (3) indifferent; (4) unpleasant; (5) very unpleasant.

DSW and TW immediately after bathing and 60 minutes after bathing. No significant changes in the scores for the degree of fatigue and comfort level in the three water groups were found between repeated measurements. The score for the degree of fatigue in TW, however, was significantly higher than that in DSW under the three conditions, and than that in SSW after bathing. No significant differences in the comfort-level scores were found among the three water groups under the three conditions.

\section{DISCUSSION}

The health effects of bathing on the human body have been studied from physical, chemical, and pharmacological points of view (Otsuka 2001). Previous studied have demonstrated that whole-body bathing in water temperatures $42^{\circ} \mathrm{C}$ or hotter causes increases in heart rate, cardiac output, and blood viscosity (Shirakura et al. 1993; Otsuka 2001). The ingredients in hot springs are absorbed into the body through the skin. It is also well known that the muriated hot springs have an advantage over mineral water in maintaining a constant body temperature (Otsuka 2001; Ueda 2001). Despite Japan being surrounded by the sea, the use of DSW in thalassotherapy has just begun. However, little has been reported regarding the health effects of DSW as the water for bathing. We therefore carried out this study to clarify whether DSW is useful to people as the water for bathing by determining the changes in lactate and pyruvate concentrations after bathing in the $42^{\circ} \mathrm{C}$ water for 10 minutes.

The reasons we selected the $42^{\circ} \mathrm{C}$ bathing condition and the 10-minutes bathing time are as follows: Japanese people can take a hot water bath for a comparatively long time, and favor a water temperature of approximately 
$42^{\circ} \mathrm{C}$ for bathing (Otsuka 2001). A previous study has determined that subjects feel pain in the $42^{\circ} \mathrm{C}$ bath after 10 minutes or longer (Tanaka et al. 1990).

Hot water bathing at $42^{\circ} \mathrm{C}$ or hotter has the effect of enhanced activity of the sympathetic nervous system (Otsuka 2001). It may also, however, have negative effects on human health such as fatigue and positive effects such as the producing a physically and psychologically active condition. A previous study has demonstrated that the rate of claimed subjective fatigue increased in a $41-42^{\circ} \mathrm{C}$ group immediately after bathing, but decreased 20 minutes later (Kiuchi et al. 1983). Very few studies of the changes in lactate and pyruvate concentrations after bathing at rest have been published, though there have been many studies of the changes after bathing in connection with exercise tolerance.

Lactate and pyruvate are critical metabolic products in the human body. Blood lactate concentration is a function of two factors: lactate production in the body and removal from blood circulation. Peripheral vasodilatation and muscle contraction are caused after taking a bath. Subsequently, blood circulation is improved and synthesis from lactate to glucose is promoted. As a result, lactate concentrations decrease.

First, we wanted to confirm whether the changes in plasma lactate and pyruvate concentrations were caused by the hemoconcentration, since hot water bathing for a long period of time can cause hemoconcentration due to the increase in diaphoresis. To explore the possibility of an interaction between bathing and hemoconcentration, we examined $\mathrm{Hb}$ concentrations before, immediately after, and $60 \mathrm{~min}$ utes after bathing. As shown in Table 2, the changes in $\mathrm{Hb}$ concentration immediately after bathing or 60 minutes after bathing among the three water groups were $1-2 \%$ at most, while those in lactate and pyruvate were $14-28 \%$. Therefore, the changes in lactate and pyruvate concentrations after bathing could be caused primarily by other factors rather than the hemoconcentration.

Peripheral vasodilatation after hot water bathing can supply the blood underneath the skin surface voluminously based on the vasoconstriction. Consequently, it can lead to a deficiency in sufficient blood volume to excrete waste products such as lactate and pyruvate. Previous studies have demonstrated that plasma lactate concentrations show a tendency to decrease after bathing compared with concentrations before bathing in the bathing-afterexercise tolerance test (Yorozu et al. 1986; Ueda and Yazaki 2000). Our study showed that lactate and pyruvate concentrations increased immediately after bathing compared with before bathing, though no significant changes were found in the DSW and SSW groups. This opposite result may be due to difference in physical condition before bathing. The lactate concentrations increased by exercise tolerance will be decreased after bathing through the promotion of synthesis from lactate to glucose. However, in the resting state, the value after bathing will increase due to the decrease in removal from blood circulation by the hemoconcentration and so on. In the bathing-afterexercise tolerance test, it is believed that the rate of decrease in lactate concentrations is higher than that of the increase after bathing. This is one of the reasons that the lactate concentration after bathing decreased. On the contrary, in the resting state, the concentrations will increase after hot water bathing and then decrease while the subject rests for 60 minutes.

It is worthy of note that significant differences in lactate and pyruvate concentrations between repeated measurements in the TW bathing were observed. Some studies of the possible association between lactate or pyruvate concentrations and fatigue (Davies et al. 1986; Dengler et al. 1996; Blomstrand and Saltin 1999; LeBlanc et al. 2002) have found that these concentrations increase after strenuous exercise 
and then rapidly decrease (Eastham 1985). Because of these findings, determinations of lactate and pyruvate concentrations are used to evaluate the strenuousness of exercise or the degree of fatigue. Significant increases in lactate concentrations after TW bathing may be related to the development of fatigue.

Both DSW and SSW contain approximately $3.5 \%$ salt (Ogawa and Fujimoto 2002). After DSW and SSW bathing, this salt remains on the skin and can prevent evaporation of sweat, thereby keeping the body temperature warmer. A previous study has examined the association between mean skin or body temperature and the concentration of seawater components, and has found a significant positive association between temperature and mineral concentrations (Shimizu et al. 1998). The differences in the amounts of sweat after bathing are probably caused between TW and DSW, or SSW. A previous study has demonstrated that the amount of sweat on the forehead after TW bathing is slightly greater than that after DSW and SSW bathing (Shimizu 2002). Human sweat normally contains approximately $15 \mathrm{mmol} /$ liter lactate (Eastham 1985). Thus, the decreased lactate concentrations in TW bathing must be at least somewhat related to the increased sweat volume.

In TW bathing, significant differences in pyruvate concentrations 60 minutes after bathing compared with immediately after bathing were also found, though the changes in pyruvate were similar to those in lactate among the three water groups. The increase in pyruvate concentrations is caused by the degradation of sugar primarily after strong exercise (Eastham 1985). Additional results regarding the change in pyruvate concentrations may be obtained from a similar exercise-tolerance study. However, we should be careful in interpreting the results regarding pyruvate concentrations, as there are many metabolic pathways in pyruvate.

Our questionnaire asked for opinions regarding thermal sensation, the degree of fatigue, and comfort level. The mean score following DSW bathing did not show a significant change in thermal sensation, while it did show a significant increase after SSW and TW bathing, and subjects reported a significant decrease in thermal sensation 60 minutes after the TW bathing. Several studies have examined the effect of warm baths on the maintenance of body temperature. They have demonstrated that minerals have moisturizing action to keep the skin surface moist (Hertig et al. 1962; Shimizu et al. 1995). Our findings support these reports, since there were no significant changes in thermal sensation scores between immediately after bathing and 60 minutes after bathing in the DSW and SSW groups, though significant differences were found with TW bathing. The heat of evaporation sequenced on the evaporation of sweat may be the cause of this difference.

To our knowledge, this study was the first to examine the changes in lactate and pyruvate concentrations after hot DSW bathing. Although additional studies are needed to support our findings, DSW appears to be the mildest water to the human body among the three kinds of water, since only in DSW were no significant changes observed in the items measured. Our next trial will be a similar study using tepid water. Previous studies have suggested that tepid water bathing has a greater effect on fatigue and physiological indexes than hot water bathing (Kiuchi et al. 1983; Nakamura et al. 1996). An experiment in tepid water would help to clarify the efficacy of DSW.

\section{Acknowledgments}

This study was supported by a Grant-in-Aid from Niigata Prefecture as a part of the Deep Sea Water Utilization Technology Research and Development Program in 2002. 


\section{References}

Abo, T., Kawate, T. \& Kumagai, K. (1981) Studies on the bioperiodicity of the immune response: 1. Circadian rhythms of human T, B and NK cell traffic in the peripheral blood. J. Immunol., 126, 1360-1363.

Blomstrand, E. \& Saltin, B. (1999) Effects of muscle glycogen on glucose, lactate, and amino acid metabolism during exercise and recovery in human subjects. J. Physiol., 514, 293-302.

Buskila, D., Abu-Shakra, M. \& Sukenik, S. (2001) Balneotherapy for fibromyalgia at the Dead Sea. Rheumatol. Int., 20, 105-108.

Davies, S.F., Iber, C. \& Path, M.J. (1986) Effect of respiratory alkalosis during exercise of blood lactate. J. Appl. Physiol., 61, 948-952.

Dengler, R., Wohlfarth, K. \& Schubert, M. (1996) Muscle fatigue, lactate, and pyruvate in mitochondrial myopathy with progressive external ophthalmoplegia. Muscle Nerve., 19, 456-462.

Eastham, R.D. (1985) Biochemical values in clinical medicine, John Wright \& Sons Ltd., Bristol.

Elkayam, O., Ophir, J. \& Yaron, M. (2000) Immediate and delayed effects of treatment at the Dead Sea in patients with psoriatic arthritis. Rheumatol. Int., 19, 77-82.

Evcik, D., Kizilay, B. \& Gokqen, E. (2002) The effects of balneotherapy on fibromyalgia patients. Rheumatol. Int., 22, 56-59.

Harari, M., Shani, J. \& Hristakieva, E. (2000) Climatotherapy of atopic dermatitis at the Dead Sea: demographic evaluation and costeffectiveness. Int. J. Dermatol., 39, 59-69.

Hardy, J.D. (1970) Thermal comfort: skin temperature and physiological thermoregulation. In: Physiological and behavioral temperature regulation, edited by J.D. Hardy, A.P. Gagge, J.A.J. Stolwijk, Charles C Thomas, Springfield, Illinois, USA, pp. 856-873.

Hertig, B.A., Riedesel, M.L. \& Belding, H.S. (1962) Time course of sweating in warm baths. In: Advance in Biology of Skin, Pergamon Press, Oxford.

Houghton, F.C. \& Yagloglou, C.P. (1923) Determining lines of equal comfort. Transactions of Am. Soc. of Heat.-Vent. Engineers, 29, 163176.

Kiuchi, T., Otsuka, S. \& Tsunenari, K. (1983) The effects of taking hot baths on some physiological indexes. Bull. Tokushima Univ., 34, 6979. (in Japanese with English summary)
LeBlanc, P.J., Parolin, M.L. \& Jones, N.L. (2002) Effects of respiratory alkalosis on human skeletal muscle metabolism at the onset of submaximal exercise. J. Physiol., 544, 303313.

Linder, M.C. (1991) Nutritional biochemistry and metabolism, 2nd ed., Elsevier, New York.

Nakamura, K., Takahashi, H. \& Tanaka, M. (1996) Effects of immersion in tepid bath water on recovery from fatigue after submaximal exercise in man. Ergonomics, 39, 257-266.

Neumann, L., Sukenik, S. \& Buskila, D. (2001) The effect of balneotherapy at the Dead Sea on the quality of life of patients with fibromyalgia syndrome. Clin. Rheumatol., 20, 15-19.

Nissen, J.B., Avrach, W.W. \& Hansen, E.S. (1998) Stengaard-Pedersen K. Increased levels of enkephalin following natural sunlight (combined with salt water bathing at the Dead Sea) and ultraviolet A irradiation. Br. J. Dermatol., 139, 1012-1019.

Ogawa, T. \& Fujimoto, T. (2002) A characteristic of the Japan Sea Proper Water. Ann. Rep. Inst. Environ. Geol. Sci., 13, 69-79. (in Japanese)

Otsuka, Y. (2001) Balneotherapy, 2nd ed., NANZANDO Co., Ltd., Tokyo. (in Japanese)

Sasaki, T. (2001) Deep Seawater, Bestsellers Publishers Co., Ltd. Tokyo. (in Japanese)

Shimizu, T., Fujishima, K. \& Oogaki, T. (1995) Changes of body temperature and heart beat response in hot sea water bathing. Health Science, 17, 103-108. (in Japanese with English summary)

Shimizu, T., Fujishima, K. \& Agishi, Y. (1998) Effects of concentration of sea water components on body temperature by bathing. $J$. Jpn. Assoc. Phys. Med. Balnel. Climatol., 61, 195-201. (in Japanese with English summary)

Shimizu, T. (2002) Thermophysiological responses to bathing in deep sea water, Seminar on deep sea water and ocean resources (Niigata).

Shirakura, T., Kubota, K. \& Tamura, K. (1993) Blood viscosity and cerebral blood flow. Jpn. J. Geriat., 30, 174-181. (in Japanese with English summary)

Sukenik, S., Giryes, H. \& Buskila, D. (1994) Treatment of psoriatic arthritis at the Dead Sea. J. Rheumatol., 21, 1305-1309.

Tanaka, M., Nakamura, K. \& Takahashi, K. (1990) Physiological and psychological responses on water temperature in bathing, The 14th sym- 
posium on Man-Thermal Environment System (Tokyo), 110-113. (in Japanese)

Tsubaki, T., Saito, H. \& Iikura, Y. (1994) Changes in histamine levels in skin chambers. Application for clinical evaluation of atopic dermatitis-report 3. Allergy, 43, 619-624. (in Japanese)

Tsuchiya, Y., Nakamura, K. \& Yamamoto, M. (2002) Subacute effects of deep-sea water from the Japan Sea on blood examination values in mice. Environ. Health Prev. Med., 7, 189-192.

Ueda, M. \& Yazaki, T. (2000) The effects of bathing using a new $\mathrm{CO}_{2}$ bath-water generator on changes of blood lactic acid density after exercise. J. Jpn. Assoc. Phys. Med. Balnel. Climatol., 63, 73-78. (in Japanese with English summary)

Ueda, M. (2001) Effects of hot springs and the applications to medical science. J. Jpn. Pharmaceut. Assoc., 53, 65-71. (in Japanese)

Winslow, C.-E.A., Herrington, L.P. \& Gagge, A.P. (1937) Relations between atmospheric conditions, physiological reactions and sensations of pleasantness. Am. J. Hyg., 26, 103-115.

Yamana, K., Sakurada, N. \& Kosugi, R. (2002) F.E.R.C. Research Report-File No. 077. <http://www.ntv.co.jp/FERC/research/ 20020113/r077.html >.

Yorozu, H., Eguchi, Y., Sunakawa, M., Kohmoto, T. \& Komoto, Y. (1986) Research for carbon dioxide bathing $\mathrm{V}$. Effects of the artificial $\mathrm{CO}_{2}$-bathing on blood lactic acid and myalgia. J. Jpn. Assoc. Phys. Med. Balnel. Climatol., 49, 89-94. (in Japanese with English summary)

Yoshida, K. (1978) Studies on the thermal comfort. J. Anthropological Society of Nippon, 86, 51-63. (in Japanese with English abstract) 